\title{
The Occurrence of Struvite (Magnesium Ammonium Phosphate Hexahydrate) in Microbial Cultures
}

\author{
By JANE BEAVON AND N. G. HEATLEY \\ From the Pathological Laboratory, Royal South Hants Hospital, Southampton \\ and the Sir William Dunn School of Pathology, University of Oxford
}

(Received 25 August 1962)

\begin{abstract}
SUMMARY
Crystals which occurred within colonies of Staphylococcus aureus growing on nutrient agar were identified as struvite (magnesium ammonium phosphate hexahydrate). Other organisms, all of which increased the $\mathrm{pH}$ value of the medium during growth, formed similar crystals.
\end{abstract}

\section{INTRODUCTION}

In 1958 one of us (J.B.) noticed that colonies of a particular strain of Staphylococcus aureus on plates which had been incubated for $24 \mathrm{hr}$. and then left in the refrigerator for several days contained well formed birefringent crystals (see Pl. 1). Preliminary investigation encouraged by the late Dr E. S. Duthie, showed that these crystals could be washed repeatedly with water without dissolution, and dissolved only slowly in dilute hydrochloric acid without evolution of gas. They contained phosphorus, and because they blackened on heating it was thought that they were probably organic. The ultraviolet absorption curve was typical of a purine or pyrimidine, the solubility also being characteristic of some of these bases; however, quantitative investigation showed that less than 1 part in 3000 of the crystals could have been purine or pyrimidine. Further tests showed that the crystals were mainly inorganic and liberated ammonia on treatment with alkali in the cold. Magnesium ammonium phosphate is said to be soluble to the extent of 1 part in $\mathbf{7 7 0 0}$ of water at room temperature, and comparison with the authentic compound showed that the crystals were almost certainly this substance. Since rigid identification of these small crystals by conventional means would have been difficult, a comparison of them with authentic magnesium ammonium phosphate by $\mathbf{X}$-ray crystallographic methods was sought. Mr H. M. Powell, F.R.S. (Department of Crystallography, University of Oxford) to whom we are deeply indebted for the comparison, reported as follows. 'A single crystal of the first sample provided, a laboratory-prepared specimen of authentic struvite which could not have contained any calcium, was mounted about a principal axis and $\mathrm{X}$-ray diffraction patterns obtained with $\mathrm{Cu} \mathrm{K} \alpha$ radiation. Both $15^{\circ}$ oscillation and zero layer Weissenberg photographs were taken. The patterns were typical of orthorhombic symmetry for a crystal of cell dimensions approximately $6.9 \times 11.1 \times 6 \cdot 1 \AA$. Nearly one hundred independent reflexions were accessible in the Weissenberg range used; some of those observed were close to the limiting Bragg angle, $\theta=90^{\circ}$. Similar Weissenberg 
photographs were taken of two crystals obtained from staphylococcal colonies on different [nutrient agar] plates. All three Weissenberg photographs showed, on superposition, complete identity of the spacings of the reflexions, and of the smallest details of the intensities of the different reflexions, including a number which were, for accidental structural reasons, of zero intensity. This constitutes a very firm identification.'

Mr Powell pointed out to us that the occurrence of struvite (magnesium ammonium phosphate hexahydrate) in peptone, tinned food, and material rich in organic matter has been reported at intervals for many years (Groth, 1910; Dana, 1946). Indeed, we later found that Robinson (1889) identified such crystals and suggested '. . . that the micro-organisms produce the ammonia from the nitrogenous organic matter in which they are growing and that it then combines with the magnesium phosphate present...'To him undoubtedly belongs the priority in this field.

While we were awaiting the results of the X-ray crystallographic investigation, the paper by Hutchison (1961) on crystals in colonies of Staphylococcus aureus appeared; it gives in detail the appearance, properties, conditions under which the crystals are formed. The crystals in his excellent photographs closely resemble our own in appearance, though they were found by him to be a complex calcium phosphate. Dr Hutchison agreed to co-operate with a view to establishing the identity or non-identity of his crystals with our own. However, he has none of his original crystals, and as he points out, crystals prepared by him, now, at least 5 years after his original observations and in a different laboratory, would not necessarily be identical with those originally isolated.

The nature of the colonial crystals might vary with the ions available in the medium derived from different agars, nutrients, or tap water. We investigated this possibility by a number of different experiments, from which we draw the following conclusions. (1) Apparently identical crystals may appear in the nutrient agar as well as, or instead of, in colonies. (2) Both types of crystal are larger and/or more numerous, the larger the amount of $\mathbf{M g}$ added (as sulphate) to the medium. (3) The addition of $\mathrm{Ca}$ (as chloride) decreases, or in higher concentration prevents, the formation of extra- or intra-colonial crystals; this is presumably due to removal of some of the phosphate as insoluble calcium phosphate, which can be detected as a slightly increased opalescence in the medium in the higher concentrations used. (4) When nutrient agars of different ionic composition are exposed to ammonia vapour large numbers of crystals form within the agar in a few minutes. They are larger in media which contain added $\mathrm{Mg}$ and scantier or absent altogether when extra $\mathrm{Ca}$ is present. (5) When the same media is re-exposed to triethylamine vapour, the agar becomes alkaline as quickly as with ammonia, but only after an hour or two are any crystals seen, and they are very scanty or, when $\mathrm{Ca}$ has been added, absent. (6) Intra-colonial crystals form more readily in rich media when colonies grow fast to a large size. Presumably the local concentration of ammonia is higher in such colonies than in smaller ones. Ammonia is readily detectable when such plates are opened, both by odour and the effect on litmus paper. (7) Organisms other than the staphylococcus, e.g. Pseudomonas pyocyanea, Proteus spp. and Escherichia coli, will form intra-colonial crystals; within any group those strains which form alkali the more readily are those in which crystals appear earlier and more abundantly. (8) Crystals were also formed by the staphylococcus in fluid 


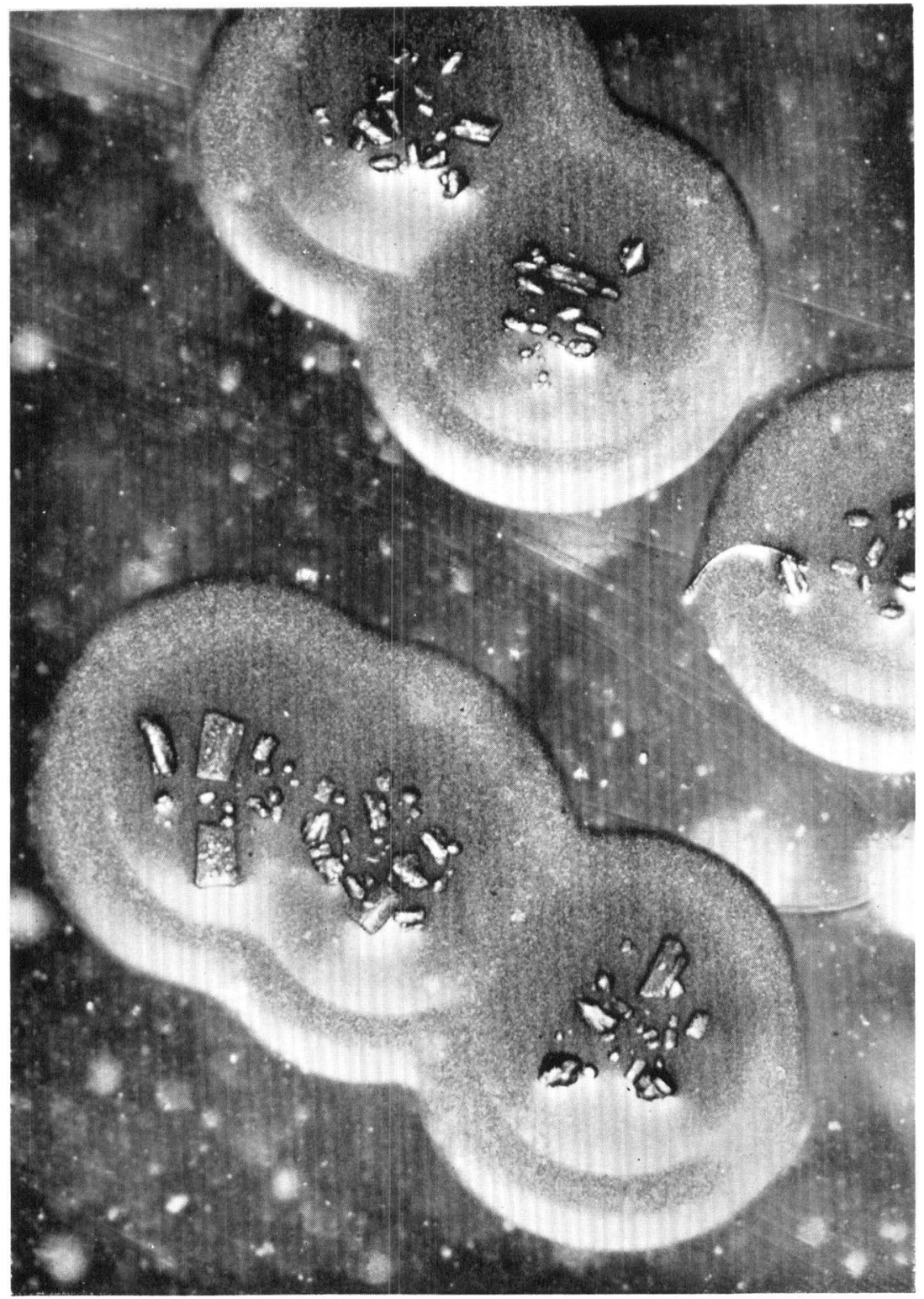



media, possibly because the organisms tended to sediment and presumably caused a higher local ammonia concentration.

The question of the identity or non-identify of our crystals with those observed by Hutchison thus remains unsettled. The fact that addition of calcium to media suppressed the formation of the typical crystals, by formation of amorphous calcium phosphate, does not rule out the possibility that a crystalline calcium phosphate could be formed under other conditions. On the other hand mere alkalinity, with or without added calcium, leads to very scanty crystals or none, suggesting that ammonium is essential for their formation.

We thank Mr Keith Tyler for taking the photograph (Pl. 1).

\section{REFERENCES}

Dana's System of Mineralogy (1946). Ed. by C. Palache, H. Berman \& C. Frondel 7th edn. London: Chapman and Hall.

Groth, P. (1910). Chemische Krystallographie, 2, 841. Leipzig: W. Engelmann.

Hutchison, J. G. P. (1961). Crystals in colonies of Staphylococcus aureus. J. Path. Bact. Bact. 82, 214.

Robinson, H. (1889). On the formation of struvite by micro-organisms. Proc. Camb. phil. Soc. 6, 360.

\section{EXPLANATION OF PLATE 1}

Colonies of Staphylococcus aureus strain $80 / 52$ on nutrient agar. Incubated at $37^{\circ}$ for 24 hr. then stored at $4^{\circ}$ for 2 weeks. 